\title{
Aporte a la cronología de chenques tardíos del lago Salitroso (Santa Cruz, Argentina)
}

\section{( Solana García Guraieb*, Rafael A. Goñi* y Rocío Guichón Fernández ${ }^{* *}$}

Recibido:

31 de marzo de 2017

Aceptado:

30 de agosto de 2017

\section{Resumen}

Se presentan dos nuevos fechados radiocarbónicos obtenidos en uno de los chenques tardíos (ca. 800-350 AP) del lago Salitroso (SAC) y sus implicaciones para las discusiones vigentes sobre prácticas mortuorias y cuestiones demográficas en el área. Se trata de SAC 1-2, un chenque primario múltiple, compuesto por un adulto femenino y 5 individuos infantiles. Dos fechados anteriores lo situaban en el orden de $c a .450$ años AP. Actualmente, se fecharon dos subadultos hallados juntos en la base del entierro, cuya posición sugería su inhumación contemporánea. Mientras uno de los individuos mantuvo el orden de los ca. 400-500 años AP (487 \pm 23 AP), el segundo presenta una fecha (709 \pm 30 AP) que se aleja más de 200 años radiocarbónicos del anterior. Esta situación genera, nuevas preguntas sobre las prácticas mortuorias y la manera en que ocurre la reutilización de las estructuras de entierro. Además, tiene implicaciones para ideas previas sobre la recurrencia de episodios de simultaneidad de muerte de subadultos de diversas edades como explicación posible para las "anomalías" identificadas en el perfil de mortalidad de los chenques tardíos. Este caso alerta sobre la necesidad de refinar aún más el detalle de la cronología de los entierros del lago Salitroso.

\section{Contribution to the chronology of late Chenques from Lake Salitroso (Santa Cruz, Argentina)}

\footnotetext{
Abstract

In this article, two new radiocarbon dates from a late chenque (ca. 800-350 AP) at Lake Salitroso (SAC) are presented. These dates have implications for current discussions concerning mortuary practices and demography in the region. The case discussed is chenque SAC 1-2, a multiple individual, primary burial, containing one female adult and 5 infants. Two prior radiocarbon dates date this burial to $c a .450 \mathrm{BP}$. The two new radiocarbon dates were from two sub-adults placed next to each other at the base of

* Instituto Nacional de Antropología y Pensamiento Latinoamericano (INAPL) - CONICET. 3 de febrero 1378 (CP C1426BJN) Ciudad Autónoma de Buenos Aires. E-mail: solanagg@gmail.com; rafaelagustingoni@gmail.com

** Instituto de Investigaciones Arqueológicas y Paleontológicas del Cuaternario (INCUAPA), Facultad de Ciencias Sociales, Universidad Nacional del Centro de la Provincia de Buenos Aires (UNICEN) - CONICET. Av. Del Valle 5737 (CP B740oJWI) Olavarría, Buenos Aires, Argentina. E-mail: rocioguichon@hotmail.com
}

Palabras clave

Registro mortuorio Cronología Demografía Holoceno tardío Patagonia

\section{Keywords}

Mortuary record Chronology Demography Late Holocene Patagonia 
the burial. Their positions suggest contemporaneous inhumation. Yet, while the date from one of the individuals ( $487 \pm 23 \mathrm{BP}$ ) was within the same chronological range of ca. 400-500 BP, the other individual yielded a date $-709 \pm 30 \mathrm{AP}-$ set approximately 200 years earlier. This chronological discrepancy raises new questions concerning mortuary practices and the way in which burial structures were reused in the region. It also has implications for existing hypotheses regarding the reoccurrence of mortality events that affected coevally sub-adults of different ages. This has been posed as a possible explanation for the abnormal pattern observed in the mortality profile of the late chenques. Our case-study warns us on the need to further refine the chronology of human burials from Lake Salitroso.

\section{Introducción}

El objetivo de este trabajo es presentar nuevos datos cronológicos recientemente obtenidos para dos individuos inhumados en chenques del Holoceno tardío de la cuenca del lago Salitroso (Santa Cruz) y evaluar las implicaciones que los mismos plantean para las discusiones vigentes en el área acerca de distintos aspectos del poblamiento humano tardío por parte de grupos cazadores-recolectores. Específicamente, se abordan las implicaciones respecto de las prácticas mortuorias y la demografía.

Iniciadas en 1997, las investigaciones en el lago Salitroso (Figura 1) han permitido recuperar una de las series más numerosas, tanto a nivel regional como nacional, de restos óseos humanos atribuibles a cazadores-recolectores del Holoceno tardío. Se han excavado con resultados positivos 37 estructuras de entierro, concentradas en un área de $200 \mathrm{~km}^{2}$, la mayoría de los cuales se encuentra dentro de la Estancia Sierra Colorada (SAC), al pie de la sierra homónima (García Guraieb, Goñi y Tessone, 2015; Goñi, Barrientos y Cassiodoro, 2000-2002). Los entierros se presentan en diversas modalidades: nichos, entierros bajo bloques (EBB) y chenques (Goñi et al., 2000-2002). En los últimos años la cronología establecida a partir de 30 fechados AMS sobre restos humanos permitió establecer tres grupos cronológicos. Los nichos y EBB, conforman un grupo de 10 estructuras con una cronología de entre $c a .2600$ y 2200 años, mientras que los chenques, corresponden a la modalidad más representada en la cuenca y conforman dos grupos: uno de chenques iniciales, formado por dos estructuras que abarcan el lapso entre $c a .1600$ y 1200 años AP y otro, de chenques tardíos, en el que se incluyeron las restantes 25 estructuras, que presenta fechados entre $\mathrm{ca} .800$ y 350 años AP (García Guraieb et al., 2015; Goñi et al., 2000-2002). Estos últimos, objeto de este trabajo, son entierros mayoritariamente primarios y múltiples, conformados en estructuras artificiales de piedra, de contorno oval o subcircular, de entre 3 y $5 \mathrm{~m}$ de diámetro y menos de $1 \mathrm{~m}$ de altura, que cubren las inhumaciones realizadas a nivel de la superficie o aprovechando depresiones someras del terreno (García Guraieb, 2010; Goñi et al., 2000-2002).

El estudio desde diferentes líneas de evidencia arqueológica y bioarqueológica de los entierros ha permitido discutir diferentes aspectos del poblamiento tardío de la cuenca del lago Salitroso (Barrientos et al., 2007; Cassiodoro, 2011; García Guraieb, 2010; García Guraieb et al., 2015; Goñi, 2000, 2010; Goñi et al., 2000-2002; Guichón Fernández, 2016; Pérez, Béguelin y Del Papa, 2004; Tessone et al., 2009; Tessone, García Guraieb, Goñi y Panarello, 2015; entre otros). En especial, el grupo de los chenques tardíos presenta diversas características compatibles con un uso residencial y relativamente estable de la cuenca para el último milenio, momento en el que, en el marco de una tendencia general hacia condiciones de mayor aridez, la cuenca baja del lago Salitroso (150-300 m s.n.m) habría presentado buenas condiciones para la habitación humana (e.g. agua, 

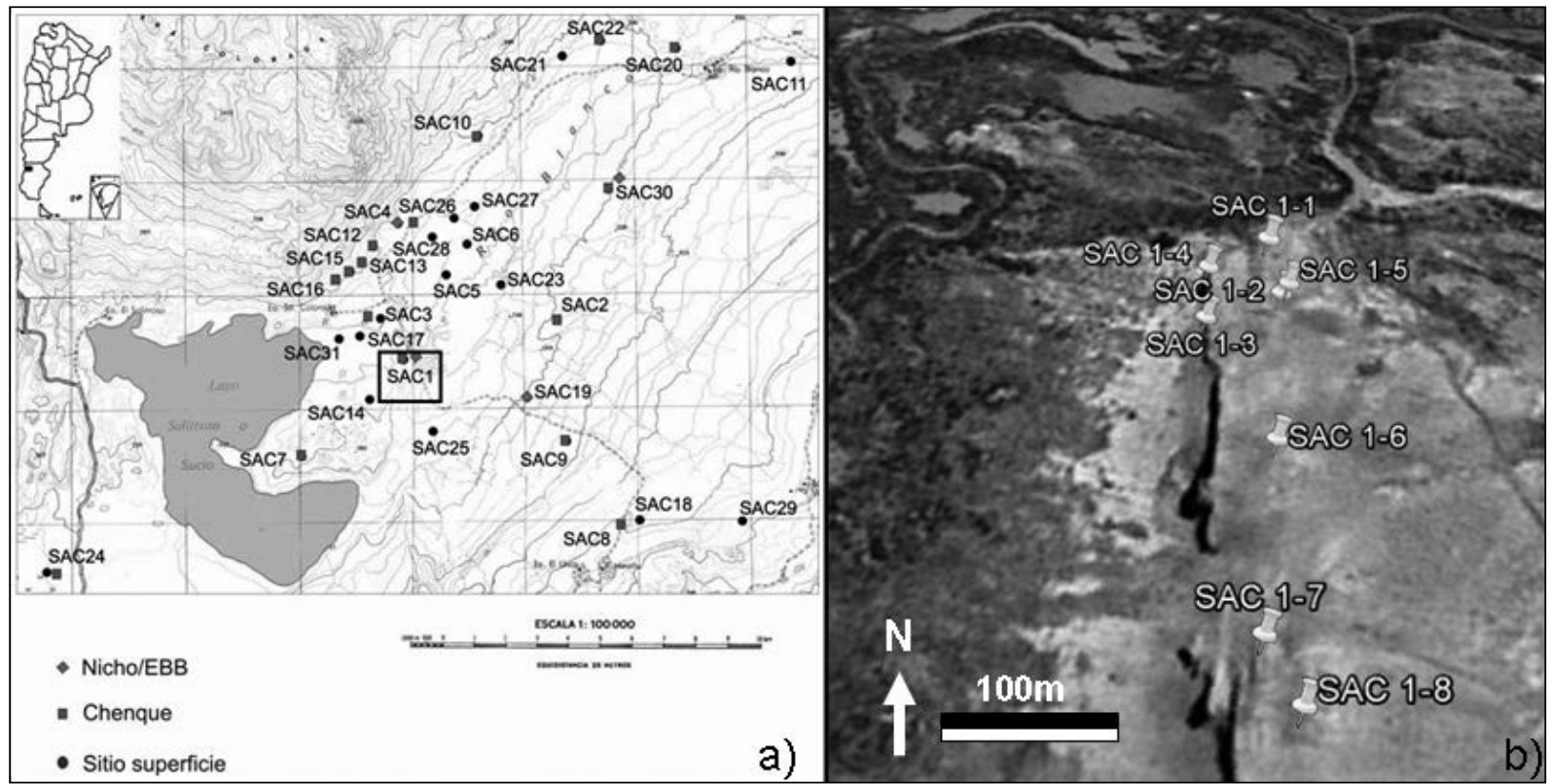

Figura 1. a) cuenca del Lago Salitroso con ubicación de sitios de entierro y a cielo abierto; b) detalle del sitio SAC 1 y sitios de entierros

leña, recursos vegetales y animales, baja carga de nieve invernal, etc.) (Goñi, 2000, 2010; Goñi et al., 2000-2002).

En este sentido, los chenques tardíos, presentan en su mayoría entierros múltiples, de cantidad de individuos y composición sexo-etaria variable. Considerando el total de individuos recuperados en ellos $(n=71)$, se observa una importante representación de adultos femeninos y subadultos menores de 10 años (Barrientos, Del Papa, Pérez y Suárez, 2004; Bernal, García Guraieb y González, 2004; García Guraieb, González y Bernal, 2007; García Guraieb et al., 2015). Esta composición así como el hecho de que se trata en su mayoría de inhumaciones primarias y extendidas, conformando un área formal de entierro, constituyen una de las evidencias que sustentan la idea de que la cuenca habría funcionado como un lugar eminentemente residencial y estable de grupos familiares (ver Barrientos, 2002).

Por su parte, los análisis de la distribución etaria de la serie de chenques tardíos han mostrado que en términos generales la alta representación de subadultos menores de 10 años es comparable con lo observado en otros perfiles osteológicos de cazadoresrecolectores y lo esperado demográficamente para estos grupos. Sin embargo, hay en la serie una representación relativa de individuos de entre 5 y 10 años mayor que la esperada. Demográficamente, lo observado en diferentes sociedades es que la mayor mortalidad entre los subadultos se registra en los individuos menores de un año, ya que es el momento de mayor vulnerabilidad biológica (Bogin, 2002; Chamberalain, 2006). Sin embargo, en la serie del lago Salitroso, dentro de la cohorte de 0-4,9 años, se identificó una menor representación relativa del subgrupo de menores de un año, lo que genera equidad en la representación entre las cohortes de 0-4,9 años y de 5-9,9 años (García Guraieb, 2010; García Guraieb et al., 2015). Recientes estudios tafonómicos evaluaron la integridad anatómica y preservación ósea de los restos de subadultos. Los resultados evidenciaron que, si bien en términos generales los subadultos de $0 \mathrm{a}$ 4,9 años presentaban una integridad menor que los mayores de 5 años, los individuos de la cohorte de 0-0,9 años no presentaban un estado de preservación diferencial (Guichón Fernández, 2016). De esta manera, su subrepresentación no podría explicarse en principio por sesgos tafonómicos. Otra de las hipótesis planteadas en trabajos previos 
(García Guraieb, 2010) fue el denominado "efecto de muestreo en la mortalidad" referido a que, en una serie osteológica de pequeño tamaño como la analizada, un mínimo evento que produjera el agrupamiento de muertes simultáneas de niños y juveniles (e.g. una epidemia, un accidente, un episodio de violencia), podría afectar la representación de estas clases etarias en relación con las otras (Paine y Boldsen, 2002). En este marco, comprender cabalmente el grado de contemporaneidad de los chenques múltiples, en especial aquellos de subadultos, es importante para avanzar en la evaluación de estas hipótesis.

Otro punto a destacar aquí por su vinculación con el caso presentado es que, desde el comienzo de las investigaciones, se identificaron casos de chenques múltiples con evidencias de reutilización. Tales evidencias refieren a individuos quemados parcial o completamente, por encima o por debajo de individuos sin evidencias de combustión, o esqueletos con signos de haber sido removidos parcialmente y restos óseos reubicados dentro del mismo chenque. Esto fue particularmente notable en el chenque SAC 1-1, en el cual se registraron al menos nueve individuos en un lapso temporal comprendido entre ca. 622 y 352 años AP, incluyendo cuentas de vidrio de origen europeo del siglo XVI (Cassiodoro y García Guraieb, 2009; Guichón Fernández, 2016). Así, en este y otros casos en la cuenca, los chenques con evidencias de reutilización quedaban claramente identificados por la remoción de restos o las evidencias de combustión y, cuando la reutilización había ocurrido en un lapso prolongado, la misma se corroboraba con los fechados radiocarbónicos obtenidos en esa estructura.

En este contexto, y con estas problemáticas en mente, se presentan a continuación los nuevos fechados disponibles para el chenque múltiple SAC 1-2 y se discuten sus implicaciones en relación con las prácticas mortuorias, la reutilización de estructuras y con las interpretaciones demográficas derivadas.

\section{Nuevos fechados para el chenque SAC 1-2}

El caso que se presenta es el del chenque SAC 1-2. Se encuentra ubicado en el sitio SAC 1 (Figura 1), que es la mayor concentración de estructuras de entierros dentro de toda el área SAC, donde se ubican otras siete estructuras, cinco de las cuales son chenques tardíos. SAC 1-2 es un entierro múltiple, con un adulto medio femenino y cinco subadultos de diferentes edades (Goñi y Barrientos, 2000). El individuo adulto (SAC 1-2-2) yacía en posición decúbito ventral con los miembros superiores e inferiores extendidos, estos últimos levemente separados y el tronco arqueado hacia el lado izquierdo respecto de los miembros inferiores. No se encontró el cráneo pero, aunque no se pudo determinar la causa de su ausencia con certeza, parece haber sido objeto de vandalismo. Este individuo habría sido ubicado boca abajo, de manera "poco cuidada" y estaba directamente por encima de los dos individuos subadultos infantiles para los cuales se presentan los nuevos fechados en este trabajo (SAC 1-2-4 y SAC 1-2-5). Estos últimos yacían en la base del entierro, en posición decúbito dorsal extendida, uno junto al otro y en la misma dirección. Los tres individuos mostraron además una completitud anatómica relativamente alta y un estado de preservación general bueno. Por su parte, el subadulto SAC 1-2-1 mostraba un grado de articulación menor y yacía parcialmente sobre los miembros inferiores del individuo SAC 1-2-2. Finalmente, los restos del individuo SAC 1-2-3 corresponden a un infantil, que se encontró apoyando parcialmente sobre las rocas que conforman la estructura de piedras del chenque. Estaba cubierto por rocas sedimentarias; presentaba mayor grado de fragmentación, menor completitud y evidencias de combustión incompleta (Goñi y Barrientos, 2000; Figura 2a). Las observaciones de campo realizadas en su momento permitían sugerir al menos tres eventos de entierro. Por un lado, la íntima relación espacial y contacto de las tres inhumaciones correspondientes al adulto SAC 1-2-2 y los dos infantiles SAC 1-2-4 y SAC 1-2-5 en la estructura sugería su depositación 


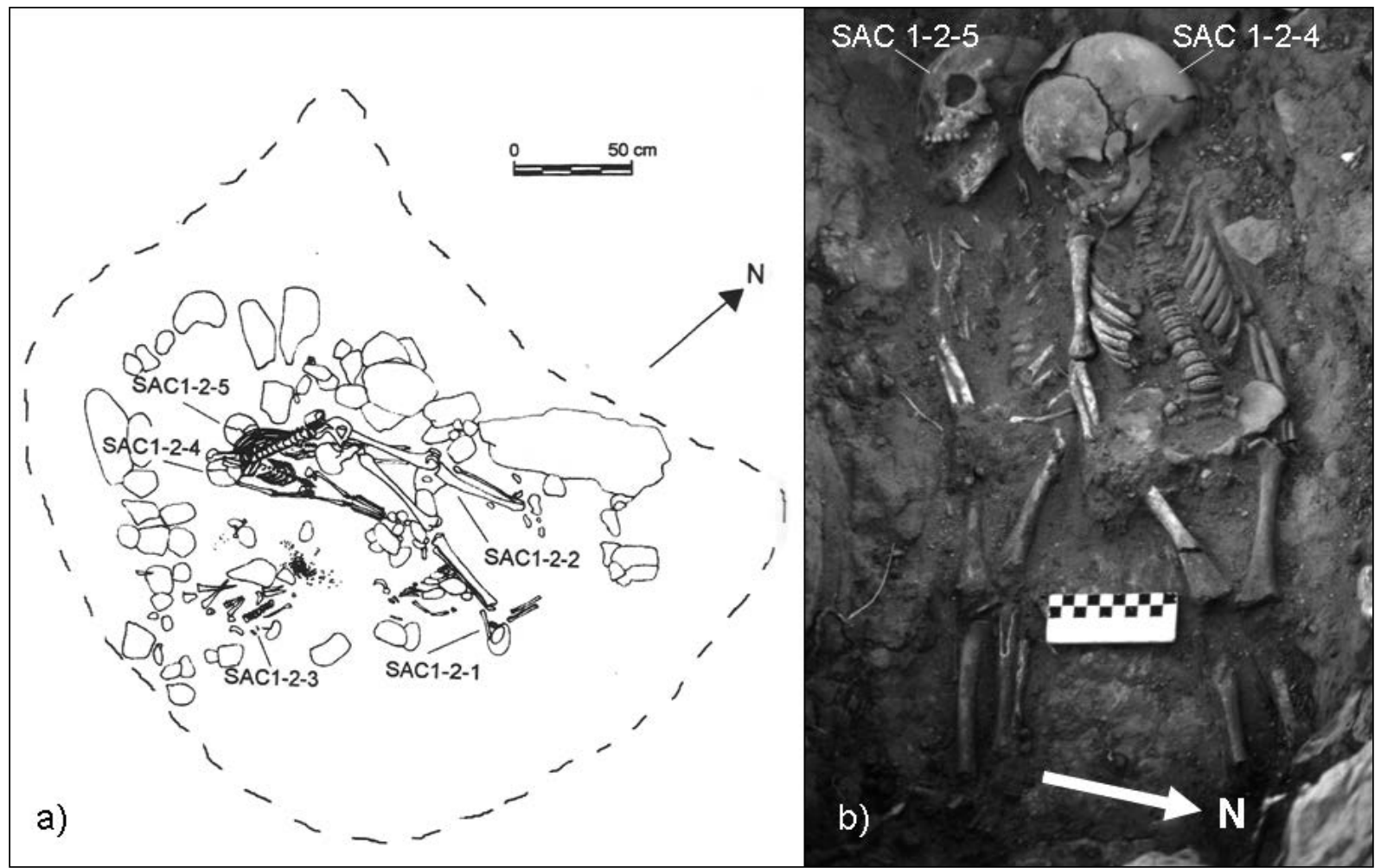

Figura 2. a) planta del chenque SAC 1-2 con superposición de los individuos SAC 1-2-2, SAC 1-2-4 y SAC 1-2-5; b) detalle de la posición de los individuos $S A C$ 1-2-4 y SAC 1-2-5.

conjunta, mientras que SAC 1-2-1 parecía corresponder a un evento posterior y SAC 1-2-3 a un tercer último episodio (Goñi y Barrientos, 2000). Los restos del individuo SAC 1-2-6 corresponden a un perinato/neonato y se encontraron mezclados con los restos generales del chenque durante las tareas de laboratorio, por lo que no pudo precisarse su correspondencia a uno de estos episodios (García Guraieb, 2010). Los primeros fechados obtenidos para este entierro (Goñi et al., 2000-2002) para los individuos SAC 1-2-1 y SAC 1-2-2 (Tabla 1), mostraban cierta contemporaneidad para al menos dos de estos eventos de inhumación identificados en el entierro. Los nuevos fechados obligan a revisar esta primera interpretación.

En la Tabla 1 se presenta la información específica de sexo, edad de cada individuo, junto con los fechados disponibles y los nuevos. Los determinación sexo-etaria de los individuos así como los métodos empleados para realizarla se han presentado en variadas oportunidades previas (Bernal et al., 2004; García Guraieb, 2010; García Guraieb et al., 2007). Todos los fechados se obtuvieron en el Laboratorio de AMS de la Universidad de Arizona. Se presenta la edad convencional y la fecha calibrada mediante el programa CALIB 7.0 (Stuiver y Reimer, 1993) empleando la curva para el Hemisferio Sur (SHe13) de Hogg et al. (2013). La Figura 3 muestra la representación gráfica de la calibración.

Como puede observarse los dos fechados obtenidos primero (SAC 1-2-1 y SAC 1-2-2) mostraban superposición de rangos. Según lo expuesto, el chenque SAC 1-2 contiene seis individuos de los cuales cinco son subadultos. Tres de los fechados radiocarbónicos, uno correspondiente al adulto (SAC 1-2-2) y otros correspondientes a dos de los subadultos (SAC 1-2-1 y SAC 1-2-4) ubican a los entierros en una condición de cierta contemporaneidad, no necesariamente simultaneidad, en el orden de los 400-500 años AP, atendiendo tanto a la fecha radiocarbónica como a su expresión calibrada (Tabla 


\begin{tabular}{|c|c|c|c|c|c|}
\hline Individuo & $\begin{array}{l}\text { Edad } \\
\text { (años) }\end{array}$ & Sexo & $\begin{array}{l}\text { Fecha Convencional } \\
\text { (años AP) }\end{array}$ & $\begin{array}{l}\text { Fecha Calibrada (2) } \\
\text { (años cal. AP) }\end{array}$ & Código de Laboratorio/Fuente \\
\hline$S_{A C} 1-2-1$ & $3 \pm 1$ & I & $418 \pm 40$ & $\begin{array}{l}425-504(p=0,56) \\
323-415(p=0,44)\end{array}$ & Goñi et al., 2000-2002 \\
\hline $\mathrm{SAC}_{1-2-2}$ & $37,5 \pm 2$ & $\mathrm{~F}$ & $389 \pm 40$ & $320-493(p=1)$ & Goñi et al., 2000-2002 \\
\hline $\mathrm{SAC}_{1-2-3}$ & $2 \pm 1$ & I & - & - & - \\
\hline $\mathrm{SAC}_{1-2-4}$ & $1,5 \pm 0,5$ & I & $487 \pm 23$ & $486-529(p=0,99)$ & AA-109156 \\
\hline$S A C_{1-2-5}$ & $2 \pm 0,6$ & I & $709 \pm 30$ & $\begin{array}{c}560-609(p=0,52) \\
624-67(p=0,48)\end{array}$ & AA-109157 \\
\hline $\mathrm{SAC}_{1-2-6}$ & $0 \pm 0,5$ & I & - & - & - \\
\hline
\end{tabular}

Tabla 1. Sexo, edad y fechados de los individuos de SAC 1-2. Referencias: $F=$ femenino; $I=$ indeterminado. Datos de sexo y edad tomados de García Guraieb (2010).

1, Figura 2 -fechados calibrados-). Sin embargo, uno de los nuevos fechados de $c a$. 700 años AP de un subadulto (SAC 1-2-5) genera nuevos interrogantes, ya que se aleja y no se superpone a dicho rango incluso considerando la expresión más amplia de su calibración (Tabla 1, Figura 3). Como se mencionó, los registros de campo y los fotográficos sugerían, en primera instancia, que los individuos SAC 1-2-4 y 1-2-5 habían sido enterrados juntos; pero esto no parece haber sucedido según el dato cronológico. La revisión de estos registros donde se puede observar un leve grado de superposición del individuo 4 sobre el 5 (Figura 2B) y una nueva observación de la colección que evidencia un grado algo mayor de meteorización y menor completitud del individuo 5 (Guichón Fernández, 2016) establecen que inicialmente hubo una apresurada interpretación de la evidencia que llevó a considerar coetáneos a dos individuos que, aunque enterrados uno al lado del otro, presentan alrededor de 200 años de diferencia. Esto no significa un problema en sí, para eso están los fechados radiocarbónicos, pero sí plantea que existió diacronía en los eventos de entierros, lo cual tiene implicaciones en las hipótesis sobre prácticas mortuorias y demografía que se venían manejando hasta el momento.

Asimismo, surge aquí otra cuestión: el chenque inicialmente fue construido para un individuo subadulto (SAC 1-2-5), posteriormente a lo cual se reutilizó, agregándole al menos otros tres individuos unos 200/300 años después. Este caso establece claramente la práctica de reutilización de estructuras para entierros, aún con varios siglos de diferencia. La explicación del porqué de este entierro inicial sin acompañamiento de adultos no está a nuestro alcance, lo destacable es que se trata de un caso especial, dado que para esta modalidad de entierro, se encuentran otros chenques con adultos, tanto en la concentración de SAC 1 (SAC 1-7 por ejemplo) como en otras concentraciones (por ejemplo, SAC 2 y SAC 4) (Goñi y Barrientos, 2000; Goñi et al., 2000-2002). Lo llamativo es que en un sector con varios entierros, el chenque SAC 1-2 fue realizado primero para el entierro de un infante al menos y luego reclamado para reutilizarlo para el entierro tanto de otros subadultos como de un adulto. Algo similar ocurre con SAC 1-6, con dos adultos medios y otro subadulto que son los primeros enterrados en dicho chenque (Goñi y Barrientos, 2000). Estas dos características: por un lado, la reutilización detectable por radiocarbono y no por evidencias de combustiones y reacomodamientos internos como en casos antes estudiados y, por el otro, el carácter fundacional de un chenque por un infante, son dos rasgos mortuorios poco frecuentes según nuestros registros anteriores.

En cuanto a la relación de este caso con la cuestión demográfica planteada anteriormente, lo observado en SAC 1-2 nos alerta sobre la interpretación de conjuntos de individuos subadultos que, al presentarse inicialmente como entierros múltiples, sugieren eventos de mortalidad sincrónica, más cuando no existen rasgos conspicuos de reutilización y la disposición espacial de los restos sugieren contemporaneidad o lapsos muy breves entre los eventos de inhumación. En este sentido cabe destacar aquí que hasta el 
Rangos de Edad Calibrada

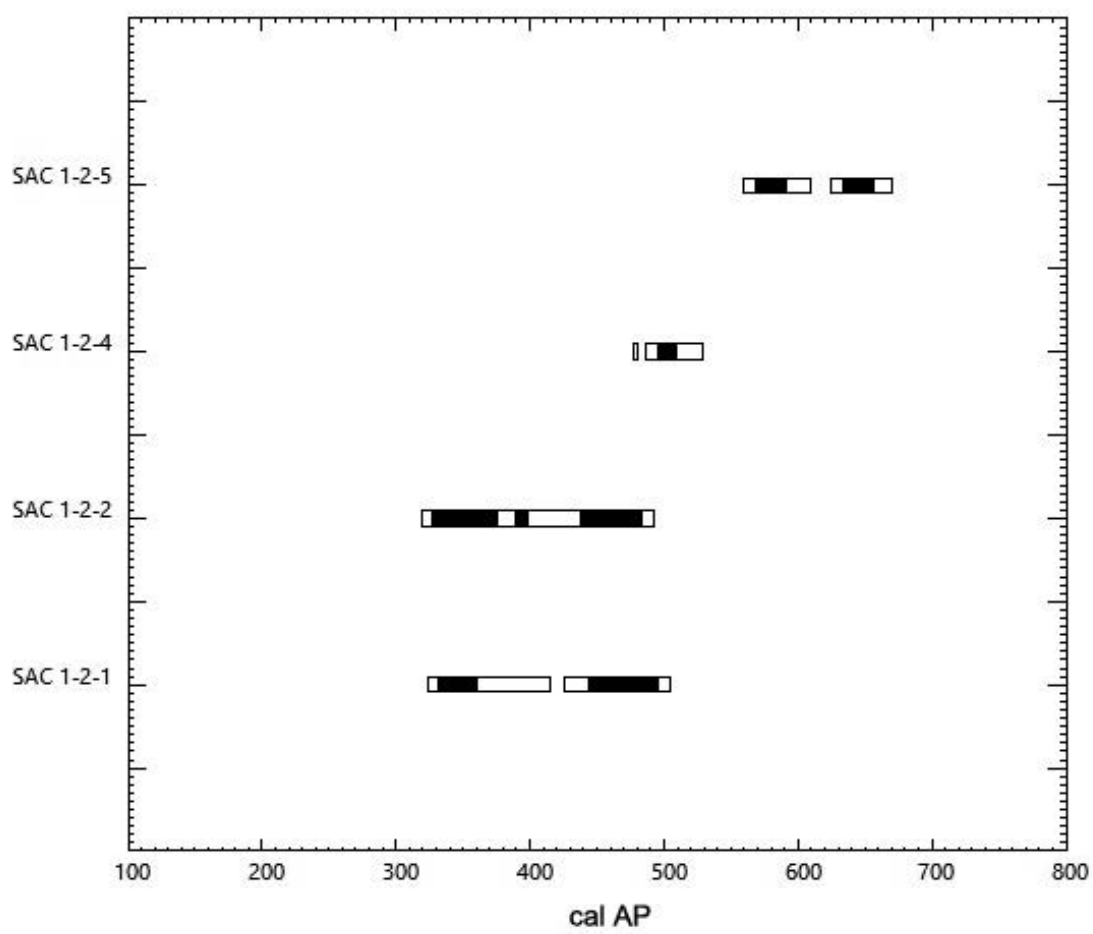

Figura 3. Representación gráfica de la calibración de los fechados de SAC 1-2 (Calib 7.o Stuiver y Reimer, 1993, Curva SHe13, Hogg et al., 2013).

momento, los chenques para los que se disponía de más de un fechado en individuos situados espacialmente próximos entre sí en la misma estructura presentaban fechados sincrónicos en términos arqueológicos (e.g. SAC 1-7-1, SAC 1-6, SAC 10-1; Figura 3; García Guraieb et al., 2015). No obstante, exceptuando el caso de SAC 10-1, en estos casos los individuos considerados son ambos adultos o un adulto en relación con un subadulto. Así, a la luz de la nueva información presentada para SAC 1-2, se hace necesario reevaluar la hipótesis planteada acerca de la recurrencia cíclica de episodios de alta mortalidad entre los subadultos de diversas edades a lo largo del lapso de ocupación de la cuenca como explicación para el patrón observado en el perfil de mortalidad de la serie del lago Salitroso. Si los entierros múltiples identificados en la cuenca, en especial aquellos conformados mayoritariamente por subadultos, (e. g. SAC 1-2, SAC 1-5, SAC 1-10, SAC 2-4, SAC 30-1, ver García Guraieb, 2010; Goñi y Barrientos, 2000; Goñi et al., 2000-2002) tienden más a la diacronía que a la sincronía, entonces, la formación de la serie osteológica de la cuenca se habría conformado principalmente de manera paulatina y acumulativa y dicha hipótesis perdería sustento dando lugar a la necesidad de una exploración más detallada de otras hipótesis propuestas, como la de la existencia de una mayor flexibilidad en las prácticas mortuorias de los individuos menores de un año (García Guraieb, 2010). Así, según hemos presentado hasta aquí, técnicas de fechados ajustadas al problema son imprescindibles para abordar hipótesis de índole demográfico.

\section{Consideraciones finales}

Se han presentado dos nuevos fechados en un chenque múltiple de seis individuos para el cual ya se contaba con información cronológica que sugería contemporaneidad en la inhumación de algunos de los individuos y un posible evento demográfico particular 
dada la cantidad de subadultos representados. El hecho de que dos nuevos fechados de individuos contiguos haya dado un resultado no esperado nos alerta sobre la necesidad de refinar la cronología de los chenques múltiples del lago Salitroso. De hecho, a pesar de contar con prácticamente un tercio de la serie osteológica fechada, la nueva información cronológica no genera redundancia en la información sino que pone en evidencia la complejidad del registro mortuorio del área, amplía la perspectiva de las discusiones vigentes y maximiza el potencial explicativo de un registro tan importante como lo es el bioarqueológico.

\section{Agradecimientos}

Las investigaciones en lago Salitroso están financiadas actualmente por el proyecto: "Variabilidad en el proceso de poblamiento humano holocénico en el centro-oeste de Santa Cruz (Patagonia Meridional)", dirigido por Rafael Goñi, UBACYT (2014-2017) 20020130100293BA y el Ministerio de Cultura de la Nación. Agradecemos a Lito García y Rosa Hoffman de Sierra Colorada y a la Comisión de Fomento de la localidad de Lago Posadas por su apoyo constante a los trabajos arqueológicos en el Lago Salitroso a los largo de los años. Nuestro especial reconocimiento también a los dos revisores anónimos por sus valiosos comentarios y sugerencias a la primera versión de este trabajo. 


\section{Q Referencias citadas}

" Barrientos, G. (2002). The archaeological analysis of death-related behaviors from an evolutionary perspective: exploring the bioarchaeological record of Early American hunter-gatherers. En J. L. Lanata y G. Martínez (Eds.), Perspectivas Integradoras entre Arqueología y Evolución. Teoría, Método y Casos de Aplicación (pp. 221-254). Olavarría: INCUAPA.

" Barrientos, G., Del Papa, M., Pérez, I. y Suarez, F. (2004). Los entierros humanos del área del lago Salitroso, provincia de Santa Cruz. En Actas del XIII Congreso Nacional de Arqueología Argentina (Tomo 4) (pp. 73-79). Córdoba: Universidad Nacional de Córdoba.

» Barrientos G., Goñi, R., Zangrando, A. F., Del Papa, M., García Guraieb, S., Arregui, M. J.y Negro, C. (2007). Human taphonomy in Southern Patagonia. En M. Gutiérrez, L. Miotti, G. Barrientos, G. Mengoni Goñalons y M. Salemme (Eds.), Taphonomy and Zooarchaeology in Argentina (pp.187-201). Oxford: British Archaeological Reports, Archaeopress.

» Bernal, V., García Guraieb, S. y González, P. (2004). Perfiles de mortalidad de las muestras de restos óseos humanos procedentes del área del Lago Salitroso (provincia de Santa Cruz). En M. T. Civalero, P. Fernández y G. Guraieb (Eds.), Contra viento y marea. Arqueología de Patagonia (pp. 362-373). Buenos Aires: Instituto Nacional de Antropología y Pensamiento Latinoamericano y Sociedad Argentina de Antropología.

"Bogin, B. (2002). The evolution of human growth. En N. Cameron (Ed.), Human Growth and Development (pp. 295-320). Amsterdam: Academic Press.

"Cassiodoro, G. (2011). Movilidad y uso del espacio de cazadores-recolectores del Holoceno tardío: estudio de la variabilidad del registro tecnológico en distintos ambientes del noroeste de la provincia de Santa Cruz. Oxford: BAR, Archaeopress.

»Cassiodoro, G. y García Guraieb, S. (2009). Análisis del registro tecnológico y osteológico de los entierros humanos del Holoceno tardío del lago Salitroso (Santa Cruz): un aporte al estudio del comportamiento mortuorio de cazadores-recolectores. En M. Salemme, F. Santiago, M. Álvarez, E. Piana, M. Vázquez y M. Mansur (Eds.), Arqueología de Patagonia: una mirada desde el último confín (pp. 613-628). Ushuaia: Utopías.

"Chamberlain, A. T. (2006). Demography in Archaeology. Cambridge: Cambridge University Press.

" García Guraieb, S. (2010). Bioarqueologia de cazadores-recolectores del Holoceno tardío de la cuenca del lago Salitroso (Santa Cruz): aspectos paleopatológicos y paleodemográficos. (Tesis Doctoral inédita), Universidad de Buenos Aires, Argentina.

» García Guraieb S., González, P. y Bernal, V. (2007). Estructura de sexo y edad de la muestra de restos humanos del Holoceno tardío del Lago Salitroso (Santa Cruz, Argentina). En F. Morello, M. Martinic, A. Prieto y G. Bahamonde (Eds.), Arqueología de Fuego-Patagonia. Levantando piedras, desenterrando huesos...y develando arcanos (pp. 367-374). Punta Arenas: CEQUA.

" García Guraieb, S., Goñi, R. A. y Tessone, T. (2015). Paleodemography of Late Holocene hunter-gatherers from Patagonia (Santa Cruz, Argentina): An approach using multiple archaeological and bioarchaeological indicators. Quaternary International, 356, 147-158.

" Goñi, R. A. (2000). Arqueología de Momentos Históricos fuera de los centros de conquista y colonización: un análisis de caso en el sur de Patagonia. En J. B Belardi, F. Carballo Marina y S. Espinosa (Eds.), Desde el País de los Gigantes. Perspectivas arqueológicas en Patagonia (pp. 283-296). Río Gallegos: Universidad Nacional de la Patagonia Austral. 
»Goñi, R. A. (2010). Cambio climático y poblamiento humano durante el Holoceno tardío en Patagonia Meridional. Una perspectiva arqueológica. (Tesis Doctoral inédita), Universidad de Buenos Aires, Argentina.

»Goñi, R. A. y Barrientos, G. (2000). Estudio de chenques en el lago Salitroso, Provincia de Santa Cruz. En J. B Belardi, F. Carballo Marina y S. Espinosa (Eds.), Desde el País de los Gigantes. Perspectivas arqueológicas en Patagonia (pp.161-175). Río Gallegos: Universidad Nacional de la Patagonia Austral.

" Goñi, R. A., Barrientos, G. y Cassiodoro, G. (2000-2002). Las condiciones previas a la extinción de las poblaciones humanas del sur de Patagonia: una discusión a partir del análisis de la estructura del registro arqueológico de la cuenca del Lago Salitroso. Cuadernos del Instituto Nacional de Antropología y Pensamiento Latinoamericano, 19, 249266.

" Guichón Fernández, R. (2016). Estudio de individuos subadultos de la cuenca del lago Salitroso: Evaluación de sesgos en la representación etaria en chenques del Holoceno tardío final (Santa Cruz). (Tesis de Licenciatura inédita), Universidad Nacional del Centro de la Provincia de Buenos Aires, Argentina.

» Hogg, A. G., Hua, Q., Blackwell, P. G., Buck, C. E., Guildersno, T. P., Heaton, T. J., Niu. M., Palmer, J. G., Reimer, P. J., Reimer, R. W., Turney, C. S. M. y Zimmerman, S. (2013). SHCal13 Southern Hemisphere Calibration, o-50,000 years cal B.P. Radiocarbon, 55(4), 1889-1903.

»Paine, R. y Boldsen, J. (2002). Linking age at death distributions and ancient population dynamics: a case study. En R. H. Hoppa y J. W. Vaupel (Eds.), Paleodemography. Age distributions from skeletal sample (pp. 169-180). Cambridge: Cambridge University Press.

»Pérez, S., Béguelin, M. y Del Papa, M. (2004). Evaluación de las relaciones biológicas regionales y extra-regionales de muestras de NO de Santa Cruz. Resultados preliminares. En M. T. Civalero, P. Fernández y G. Guraieb (Eds.), Contra Viento y Marea, Arqueología de Patagonia (pp.347-360). Buenos Aires: Instituto Nacional de Antropología y Pensamiento Latinoamericano y Sociedad Argentina de Antropología.

"Stuiver, M. y Reimer, P. J. (1993). Extended ${ }^{14} \mathrm{C}$ database and revised CALIB radiocarbon calibration program. Radiocarbon, 35, 215-230.

» Tessone, A., García Guraieb, S., Goñi, R. y Panarello, H. (2015). Isotopic evidence of weaning in hunter-gatherers from the late Holocene in Lake Salitroso, Patagonia, Argentina. American Journal of Physical Anthropology, 152, 3-11.

» Tessone, A., Zangrando, A. F, Barrientos, G., Goñi, R. A., Panarello, H. O. y Cagnoni, M. (2009). Stable isotope studies in the Salitroso lake basin (Southern Patagonia, Argentina): assessing diet of late holocene hunter-gatherers. International Journal of Osteoarchaeology, 19, 297-308. 